\title{
Caracterización Bioenergética de los Residuos de Cosecha de las Principales Especies Forestales del Noroeste de España
}

\author{
Daniel J. Vega-Nieva ${ }^{(1)^{*}}$, Marcos Fernández Lorenzo(2), Luis Ortiz Torres ${ }^{(3)}$ José J. Corral-Rivas $^{(4)^{00}}$ \\ (1) Facultad de Ciencias Forestales. Universidad Juárez del Estado de Durango. Col. Valle del Sur. C.P. \\ 34120 Durango, Dgo. México. (e-mail: danieljvn@gmail.com) \\ $(2,3)$ Escuela de Ingeniería Forestal. Universidad de Vigo. 36005 Pontevedra-España \\ (e-mail: marcosfernandez@edu.xunta.es; lortiz@uvigo.es) \\ (4) Instituto de Silvicultura e Industria de la Madera. Ciudad Universitaria C.P. 34120 Durango, Dgo.-México \\ (e-mail: jcorral@ujed.mx) \\ * autor a quien debe ser dirigida la correspondencia
}

Recibido Dic. 19, 2014; Aceptado Feb. 11, 2015; Versión final Mar. 31, 2015, Publicado Ago. 2015

\section{Resumen}

Los residuos de cosecha las principales especies forestales del Noroeste de España fueron caracterizados como biocombustible en base a su poder calorífico y contenido de cenizas. Se encontraron diferencias significativas en el poder calorífico superior anhidro (PCS) de las especies estudiadas, distinguiéndose cuatro grupos, de mayor a menor PCS: P.pinaster, $P$. radiata, $P$. sylvestris y B. celtyberica $>A$. melanoxylon, $S$. atrocynerea, $P$. avium y $P$. euramericana $>E$. globulus, $F$. sylvatica, $F$. excelsior y $Q$. robur $>C$. sativa y $A$. pseudoplatanus. En base a su poder calorífico neto a una humedad de $25 \%$, las siete primeras especies serían aptas para la producción de astillas A1. A una humedad del 10\%, sólo las cinco primeras especies seríian aptas para la producción de péllet A1. Según su contenido de cenizas, los restos forestales de las especies analizadas serían aptas para la producción de astillas o pélets de categoría A2 o B.

Palabras clave: poder calorífico, cenizas, astilla, calidad biomasa, bioenergía forestal.

\section{Bioenergetic Characterization of the Harvest Residues of the Main Forest Species of the NW of Spain}

\begin{abstract}
Harvesting residues of main forest species of northwestern Spain were characterized as biofules based on their heating value and ashes content. The studied species showed significant differences in their anhidrous Higher Heating Value (HHV). They were grouped in four groups from highest to lowest HHV: P.pinaster, $P$. radiata,$P$. sylvestris y $B$. celtyberica $>A$. melanoxylon, $S$. atrocynerea, $P$. avium y $P$. euramericana $>E$. globulus, F. sylvatica, F. excelsior y $Q$. robur $>C$. sativa y $A$. pseudoplatanus. Based on their lower heating value at a moisture of $25 \%$, the seven first species would be appropriate for the production of $A 1$ quality chips. At a moisture content of $10 \%$, only the five first species would be appropriate for A1 pellet production. Based on their ashes content, the forest residues of most of the analyzed species could be used for the production of $\mathrm{A} 2$ or $\mathrm{B}$ quality chips and pellets.
\end{abstract}

Keywords: heating value, ashes, biomass quality, forest bioenergy 


\section{INTRODUCCIÓN}

El interés en la combustión de biomasa ha crecido exponencialmente en las últimas décadas, como una importante estrategia de lucha contra el cambio climático mediante la producción de calor y electricidad renovables (e.g. Ortiz, 2006, Vega-Nieva et al., 2010a, 2010b, Viana et al., 2010,; Mendoza et al., 2012; García et al., 2013; Gómez et al., 2012, 2013, Fernández-Puratich et al., 2014; García et al., 2014a, 2014b). El Noroeste de España supone el 50\% del volumen maderable del país, habiéndose establecido un potencial de generación de $100 \mathrm{MW}$ anual a partir de residuos forestales (e.g. Dopazo et al., 2008, 2009, Vega et al., 2010). A pesar de este potencial de energía biomásica, los estudios de calidad de biomasa en la región son relativamente limitados (Nunez-Regueira, 1996, 1997, 1999, 2004, Pérez et al., 2008, Gómez et al. 2014). Los estudios disponibles se han centrado en la variación estacional de poder calorífico y cenizas de especies arbóreas y matorrales (Nunez-Regueira, 1996, 1997, 1999, 2004, Pérez et al., 2008), no existiendo sin embargo ningún estudio en la región que haya realizado un análisis estadístico de la variación de estos parámetros entre las principales especies arbóreas, similares a los trabajos realizados en otras regiones de España (Elvira y Hernando, 1989, Gillon et al., 1997) o en Portugal (Lousada et al., 2010, Telmo y Lousada, 2011a, 2011b).

Las reciente normas de calidad de astilla EN 14961-4 y la certificación de pélets EN-Plus establecen los requisitos de calidad de las astillas y pélets forestales para su combustión, definiendo categorías en función de su contenido de humedad, contenido de cenizas y poder calorífico neto, según se detalla en la tabla 1. La Tabla muestra los valores de humedad, cenizas y poder calorífico neto a humedad de recepción para las categorías A1, A2 y B de astillas y pélets según las norma europeas EN 14961-4 y EN 14961-2 (certificado En-Plus) de control de calidad de astillas pélets. En la tabla, $\mathrm{M}$ es la humedad (\% base húmeda), A son las cenizas (\% en base seca), Q es el poder calorífico neto a humedad de recepción ( $\mathrm{MJ} / \mathrm{kg})$. y A1, A2 y B son las categorías de astillas y pélets según las norma europeas EN 14961-4 y EN 14961-2

Tabla 1: Valores de humedad, cenizas y poder calorífico neto a humedad de recepción para astillas y pélets

\begin{tabular}{|c|c|c|c|c|c|}
\hline \multirow{2}{*}{$\begin{array}{l}\text { Clase de propiedad } \\
\text { Método de análisis }\end{array}$} & \multirow{2}{*}{ Unidades } & \multirow{2}{*}{ Biocombustible } & \multicolumn{3}{|c|}{ Categoría (EN 14961) } \\
\hline & & & $A 1$ & $A 2$ & $B$ \\
\hline \multirow{4}{*}{ Humedad, M } & \multirow{4}{*}{$\%$ en recepción } & \multirow{2}{*}{ Pélet (EN 14961-2) } & $\underline{\mathrm{M} 10}$ & $\underline{\mathrm{M} 10}$ & $\underline{\mathrm{M} 10}$ \\
\hline & & & $<10 \%$ & $<10 \%$ & $<10 \%$ \\
\hline & & \multirow{4}{*}{ Astilla (EN 14961-4) } & $\underline{\mathrm{M} 10}$ & M35 & A esnecificar \\
\hline & & & $<10 \%$ & & 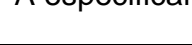 \\
\hline \multirow{2}{*}{ EN 14774-1\&2 } & \multirow{2}{*}{ (\% base húmeda) } & & M25 & \multirow{2}{*}{$<35 \%$} & \multirow{2}{*}{$>35 \%$} \\
\hline & & & $<25 \%$ & & \\
\hline \multirow{3}{*}{ Cenizas, A } & \multirow{4}{*}{$\%$ base seca } & \multirow{2}{*}{ Pélet (EN 14961-2) } & $\underline{\mathrm{A} 0,7}$ & $\underline{\mathrm{A} 1,5}$ & $\underline{\mathrm{A} 3,0}$ \\
\hline & & & $<0,7 \%$ & $<1,5 \%$ & $<3,0 \%$ \\
\hline & & \multirow{2}{*}{ Astilla (EN 14961-4) } & $\underline{\mathrm{A} 1,0}$ & $\underline{\mathrm{A} 1,5}$ & $\underline{\mathrm{A} 3,0}$ \\
\hline \multirow[t]{2}{*}{ EN 14775} & & & $<1,0 \%$ & $<1,5 \%$ & $<3,0 \%$ \\
\hline & & \multirow{2}{*}{ Pélet (EN 14961-2) } & $\underline{Q} 16.5$ & $\underline{Q 16.3}$ & Q16.0 \\
\hline \multirow{2}{*}{ Poder Calorífico Neto, Q } & \multirow{2}{*}{$\begin{array}{l}\text { a humedad de } \\
\text { recepción }\end{array}$} & & $16.5<Q<19$ & $16.3<Q<19$ & $16.0<Q<19$ \\
\hline & & \multirow{2}{*}{ Astilla (EN 14961-4) } & $\underline{Q} 13.0$ & $\underline{Q 11.0}$ & $\underline{Q<11.0}$ \\
\hline EN 14918 & $(\mathrm{MJ} / \mathrm{Kg})$ & & $>13.0$ & $>11.0$ & A especificar \\
\hline
\end{tabular}

Estos parámetros son claves para definir la calidad de los combustibles biomásicos. Por un lado, el poder calorífico es la cantidad de energía desprendida por un $\mathrm{kg}$ de combustible al quemarse, variando con la composición y humedad de las distintas especies (Ortiz, 2006). Por otro lado, el porcentaje de cenizas indica la cantidad de materia sólida no combustible por kilogramo de material, siendo un parámetro clave para predecir problemas en la combustión en las calderas, incluyendo (Ortiz, 2006): 1) la obstrucción de los ventiladores de la parrilla; 2) los sistemas automáticos de limpieza de quemadores y los intercambiadores pueden resultar dañados; 3) se generan más productos inquemados que hay que eliminar frecuentemente; 4) los automatismos, sondas, etc. pueden resultar dañados; 5) los alquitranes condensados en chimeneas pueden afectar a los electroventiladores de salida de humos.

Dentro del proyecto Europeo del VII Marco SilvaPlus, se buscó analizar las características de las principales especies forestales del noroeste de España y clasificar su calidad como biocombustible sólido en base a las normas de calidad europeas vigentes. El presente trabajo resume los resultados de caracterización 
bioenergética de residuos de corta de las 14 principales especies forestales del noroeste de España en base a su poder calorífico neto y contenido de cenizas, así como su categorización según las normas europeas de calidad como materia prima para la producción de astilla o pélet.

\section{MATERIALES Y MÉTODOS}

Se tomaron muestras representativas de residuos forestales de cosecha en el noreste español, incluyendo ramas, ramillas y hojas, de las siguientes especies arbóreas: Pinus pinaster, Pinus radiata, Pinus sylvestris, Betula celtyberica, Acacia melanoxylon, Salix atrocynerea, Prunus avium, Populus euramericana, Eucalyptus globulus, Fagus sylvatica, Fraxinus excelsior, Quercus robur, Castanea sativa y Acer pseudoplatanus.

El estudio se realizó en 14 cortas realizadas en plantaciones privadas en la provincia de Pontevedra, en el Noroeste de España. Los aprovechamientos consistieron en el apeo y desramado en monte mediante cosechadora de los pies en cortas finales a hecho. Los árboles desramados fueron transportados a planta con autocargador, y los restos del desramado se mantuvieron en monte en las áreas de trabajo de la cosechadora. La toma de muestras se centró sobre los restos de cosecha (ramas y hojas), recogidos en el mes posterior a cada corta, realizadas en los meses de junio a agosto. Se tomaron 10 muestras por especie siguiendo la norma de muestreo UNE-EN 14778-1:2013, cubriendo el rango diamétrico presente en los restos de cosecha para cada especie. Las muestras fueron transportadas en bolsas herméticas al laboratorio. En el laboratorio, se procedió al astillado y molienda de cada muestra a $1 \mathrm{~mm}$ mediante un molino Restch y se tomaron réplicas representativas para análisis de cada muestra según la norma de preparación de muestras UNE-EN 14780: 2012. Se realizaron tres réplicas de cada muestra.

El contenido de humedad fue determinado según la norma UNE-EN 14774:2010 mediante secado en estufa a $105^{\circ} \mathrm{C}$ hasta alcanzar peso constante. La determinación del contenido de cenizas (en base seca) se llevó a cabo a por combustión de $1 \mathrm{~g}$ de biomasa molida a $1 \mathrm{~mm}$ en un crisol de porcelana en una mufla a $550{ }^{\circ} \mathrm{C}$ $\pm 10^{\circ} \mathrm{C}$ durante $5 \mathrm{~h}$ siguiendo la norma CEN EN 14775: 2011. El porcentaje de cenizas se calculó como (Eq.1):

$A(\%)=$ Peso de cenizas $\left(550^{\circ}\right) /$ Peso de biomasa $\times 100$

El poder calorífico superior (PCS), de la biomasa a volumen constante en base seca se determinó en un calorímetro siguiendo la CEN EN 14918: 2009. Para ello, se pesó $1 \mathrm{~g}$ de muestra molida y se depositó en un crisol metálico de $30 \mathrm{~mm}$ de diámetro y $20 \mathrm{~mm}$ de profundidad. El crisol con la muestra fue introducido en una bomba calorimétrica, que fue depositada en el interior de un vaso del calorímetro, recipiente metálico en el que se introduce la bomba completamente recubierta por agua y recubierto por una camisa de agua o termostato, con un espacio de aire de aproximadamente $10 \mathrm{~mm}$ separando el calorímetro y el termostato. Se registró la temperatura con un sensor de temperatura, capaz de medir temperaturas con una resolución de 0,001 K. El PCS se calculó en base a la (Eq.2):

$$
q_{v, g r, d}=q_{v, g r} x \frac{100}{\left(100-M_{a d}\right)}
$$

En esta ecaucion $q_{v, g r, d}$ es el poder calorífico superior a volumen constante del combustible (sin humedad) seca, en julios por gramo; $M_{a d}$ es la humedad en la muestra de análisis, en porcentaje en masa; y $q_{v, g r}$ es el poder calorífico superior a volumen constante del combustible como se analiza, en julios por gramo.

El poder calorífico inferior en base seca se calculó como (Eq.3) :

$$
q_{p, n e t, d}=q_{v, g r, d}-212,2 \times w(H)_{d}-0,8 \times\left[w(O)_{d}+w(N)_{d}\right]
$$

En esta ecuación, $q_{p, n e t, d}$ es el valor calorífico inferior en base seca a presión constante, en julios por gramo, del biocombustible; $q_{v, g r, d}$ es el poder calorífico superior en base seca, en julios por gramo, del biocombustible; $w(H)_{d}$ es el contenido de hidrógeno, en porcentaje en masa, del biocombustible sin humedad (seco); $w(O)_{d}$ es el contenido de oxígeno, en porcentaje en masa del biocombustible sin humedad; $w(N)_{d}$ es el contenido de nitrógeno, en porcentaje en masa, del biocombustible sin humedad.

El poder calorífico inferior a presión constante a un contenido de humedad requerido w\%, o poder calorífico neto $(Q)$ se calculó como (Eq.4): 


$$
q_{p, n e t, a r}=q_{p, n e t, d} *\left(1-0.01 M_{a r}\right)-24.43 * M_{a r}
$$

Donde $q_{p, \text { red,ar }}$ es el poder calorífico inferior a presión constante, en julios por gramo, del biocombustible con contenido de humedad tal como se recibió $M_{a r}$ o poder calorífico neto $(\mathrm{Q})$; $q_{p, \text { red,d }}$ es el poder calorífico inferior a presión constante en base seca, en julios por gramo, del biocombustible; $M_{a r}$ es el contenido de humedad en que se recibieron [w\%]; 24.43 es el factor de corrección de la entalpía de vaporización (presión constante) para el agua (humedad) a $25^{\circ} \mathrm{C}$ [ $\mathrm{J} / \mathrm{g}$ por $1 \%$ en peso de humedad].

Los valores de PCS y cenizas fueron analizados mediante el siguiente modelo estadístico (Eq. 5):

$$
Y_{i}=\mu+S_{i}+\varepsilon_{i}
$$

Donde: $Y_{i}$ es el promedio de la variable analizada (PCS o cenizas) para la especie "i", $\mu$ es la media global, $S_{i}$ es el efecto aleatorio de la especie "i", $\varepsilon_{i}$ es el error experimental.

Los modelos fueron analizados mediante la función PROC GLM del paquete estadístico SAS 9.0. En caso de detectarse diferencias significativas en el análisis de varianza GLM, se realizó un test de Tukey de comparación de medias, considerándose un nivel de $\mathrm{p}<0.05$ para detectar diferencias significativas entre especies.

\section{RESULTADOS Y DISCUSIÓN}

Los resultados de poder calorífico superior (PCS) y poder calorífico neto $(\mathrm{Q})$ a humedades de 25 y $10 \%$ se muestran en la tabla 2. Tambien se muestra la categoría en base a los valores de $Q$ a estos valores de humedad para astilla y pélet según la normas EN 14961-4 y EN 14961-2, respectivamente. Se muestran las desviaciones estándar del PCS en paréntesis. En la tabla se muestra: el poder calorífico superior anhidro (PCS H=0\%) $(\mathrm{KJ} / \mathrm{kg})$, el poder calorífico neto $(\mathrm{Q})$ a humedades de 25 y $10 \%(\mathrm{KJ} / \mathrm{kg})$ y las categorías en base a Q según las normas EN 14961-4 y EN 14961-2 (certificado EN-Plus) para los valores de humedad de 25 y $10 \%$ para astillas y pélets, respectivamente. El análisis de varianza GLM detectó diferencias significativas en el PCS enentre especies. Las letras en cursiva denotan valores significativamente diferentes de PCS detectados mediante el test de Tukey a una $p<0.05$.

Tabla 2: Poder calorífico superior anhidro, poder calorífico neto y categorías

\begin{tabular}{|l|l|l|l|l|l|}
\hline & $\begin{array}{l}P C S \\
(H=0 \%)(K J / K g)\end{array}$ & $\begin{array}{l}Q \\
(H=25 \%) \\
(K J / K g)\end{array}$ & $\begin{array}{l}\text { Categoría astilla } \\
(H=25 \%) \\
(E N ~ 14961-4)\end{array}$ & $\begin{array}{l}Q \\
(H=10 \%) \\
(K J / K g)\end{array}$ & $\begin{array}{l}\text { Categoría } \\
\text { pélet }(H=10 \%) \\
(E N 14961-2)\end{array}$ \\
\hline Pinus pinaster & $20955( \pm 902)$ a & 14111 & $\mathrm{~A} 1(\mathrm{Q} 13.0)$ & 17423 & $\mathrm{~A} 1(\mathrm{Q} 16.5)$ \\
\hline Pinus radiata & $20674( \pm 730) \mathrm{ab}$ & 13900 & $\mathrm{~A} 1(\mathrm{Q} 13.0)$ & 17170 & $\mathrm{~A} 1(\mathrm{Q} 16.5)$ \\
\hline Pinus sylvestris & $20645( \pm 434) \mathrm{ab}$ & 13879 & $\mathrm{~A} 1(\mathrm{Q} 13.0)$ & 17144 & $\mathrm{~A} 1(\mathrm{Q} 16.5)$ \\
\hline Betula celtyberica & $20325( \pm 698) \mathrm{ab}$ & 13639 & $\mathrm{~A} 1(\mathrm{Q} 13.0)$ & 16856 & $\mathrm{~A} 1(\mathrm{Q} 16.5)$ \\
\hline Acacia melanoxylon & $20189( \pm 475) \mathrm{b}$ & 13537 & $\mathrm{~A} 1(\mathrm{Q} 13.0)$ & 16734 & $\mathrm{~A} 1(\mathrm{Q} 16.5)$ \\
\hline Salix atrocynerea & $19649( \pm 717) \mathrm{bc}$ & 13132 & $\mathrm{~A} 1(\mathrm{Q} 13.0)$ & 16248 & $\mathrm{~B}(\mathrm{Q} 16.0)$ \\
\hline Prunus avium & $19602( \pm 472) \mathrm{bc}$ & 13096 & $\mathrm{~A} 1(\mathrm{Q} 13.0)$ & 16205 & $\mathrm{~B}(\mathrm{Q} 16.0)$ \\
\hline Populus euramericana & $19352( \pm 257) \mathrm{bc}$ & 12909 & $\mathrm{~A} 2(\mathrm{Q} 11.0)$ & 15980 & $<16.0$ \\
\hline Eucalyptus globulus & $19335( \pm 867) \mathrm{c}$ & 12896 & $\mathrm{~A} 2(\mathrm{Q} 11.0)$ & 15965 & $<16.0$ \\
\hline Fagus sylvatica & $19179( \pm 402) \mathrm{cd}$ & 12779 & $\mathrm{~A} 2(\mathrm{Q} 11.0)$ & 15825 & $<16.0$ \\
\hline Fraxinus excelsior & $19143( \pm 387) \mathrm{cd}$ & 12752 & $\mathrm{~A} 2(\mathrm{Q} 11.0)$ & 15792 & $<16.0$ \\
\hline Quercus robur & $18776( \pm 640) \mathrm{cd}$ & 12477 & $\mathrm{~A} 2(\mathrm{Q} 11.0)$ & 15462 & $<16.0$ \\
\hline Castanea sativa & $18298( \pm 983) \mathrm{d}$ & 12118 & $\mathrm{~A} 2(\mathrm{Q} 11.0)$ & 15032 & $<16.0$ \\
\hline Acer $p s e u d o p l a t a n u s$ & $18123( \pm 319) \mathrm{d}$ & 11987 & $\mathrm{~A} 2(\mathrm{Q} 11.0)$ & 14874 & $<16.0$ \\
\hline
\end{tabular}

Los valores medidos y rangos de variación son similares a los encontrados para algunas de estas especies en la región de estudio: así Núñez-Regueira $(1996,1997,1999)$ encontró para el poder calorífico de P.pinaster rangos de $20.500-19.500 \mathrm{KJ} / \mathrm{kg}$ y valores más reducidos, en los rangos de $17.500-18.500 \mathrm{KJ} / \mathrm{kg}$, para especies de frondosas como Q. robus o C. Sativa. Valores en el rango de 17.000 a $19.000 \mathrm{KJ} / \mathrm{kg}$ fueron reportados por Pérez et al. (2008) para distintas especies de eucalipto en el noroeste peninsular, coincidiendo con los resultados del presente estudio. Los valores observados son asimismo similares a los reportados para algunas de estas especies en los estudios en distintas regiones de España de Elvira y 
Hernando (1989), Guillón et al. (1997), y Madrigal et al. (2011) o Lousada et al. (2010) y Telmo y Lousada (2011a, 2011b) en el norte de Portugal.

El análisis de varianza GLM reveló cuatro grupos de especies en base a su PCS anhidro. Las especies de valores más altos de PCS, superiores a los $20.000 \mathrm{KJ} / \mathrm{jkg}$, fueron las tres especies de Pinus, B. celtyberica $y$ A. melanoxylon. con el resto de las frondosas mostrando valores más reducidos de PCS. Estas cinco especies, junto con $S$. atrocinerea y $P$. avium, serían catalogadas como astilla A1 a un nivel de $25 \%$, máximo nivel de humedad admisible para esta categoría según la norma EN 14961-4, mientras que el resto de especies se clasificarían como astillas A2 en base a un poder calorífico neto inferior a $13.000 \mathrm{KJ} / \mathrm{kg} \mathrm{a}$ esta humedad. Con respecto al potencial para producción de pélets, tan sólo las cinco especies de mayor poder calorífico podrían ser utilizadas al $100 \%$ para la fabricación de pélet $\mathrm{A} 1$ a un nivel de humedad $<10 \%$ en base a su poder calorífico neto a esta humedad, Salix y Prunus contarían con un valor de $Q$ correspondiente a un pélet clase $\mathrm{B}, \mathrm{y}$ el resto de las frondosas necesitarían ser mezcladas con especies de poder calorífico más elevado para la fabricación de pélets EN-Plus.

Varios estudios han encontrado valores de PCS más elevado en coníferas que en las frondosas en la región de estudio: así, Lousada et al. (2010) o Telmo y Lousada (2011a,b), encontraron valores de PCS más alto en distintas especies de Pinus comparados con frondosas nativas y autóctonas en el Norte de Portugal. Similarmente Elvira y Hernando (1989) o Guillon et al. (1997) reportaron valores más elevados para coníferas que para frondosas en el estudio de varias especies arbóreas y de matorrales en España. A nivel global, varios estudios a nivel global han corroborado un mayor poder calorífico en especies de coníferas que frondosas, siendo a tribuido a sus mayores contenidos de carbono, mayores contenidos de lignina y mayores contenidos de extractivos en las resinas (e.g. Demirbas et al., 1997, Channiwala, 2001; Sheng y Azevedo, 2005; Friedl et al., 2005; Lousada et al., 2010; Telmo et al., 2010; Garcia et al., 2014a, 2014b).

La figura 1 muestra el poder calorífico superior anhidro $(\mathrm{KJ} / \mathrm{kg})$ promedio e intervalos de confianza de las 14 especies analizadas. El valor promedio se muestra como una línea horizontal en negrita, las barras verticales representan el intervalo de confianza definido por la desviación estándar de cada especie. Letras entre paréntesis diferentes denotan diferencias significativas a un nivel de $p<0.05$. Los contenidos de ceniza promedios y su desviación estándar se muestran en la tabla 3.:La figura 2 muestra el contenido de cenizas (porcentaje en base seca) promedio e intervalos de confianza de las 14 especies analizadas. El valor promedio se muestra como una línea horizontal en negrita, las barras verticales representan el intervalo de confianza definido por la desviación estándar de cada especie. Cenizas (\% b.s.): contenido de cenizas obtenidas a $550^{\circ} \mathrm{C}$, en porcentaje en base seca.

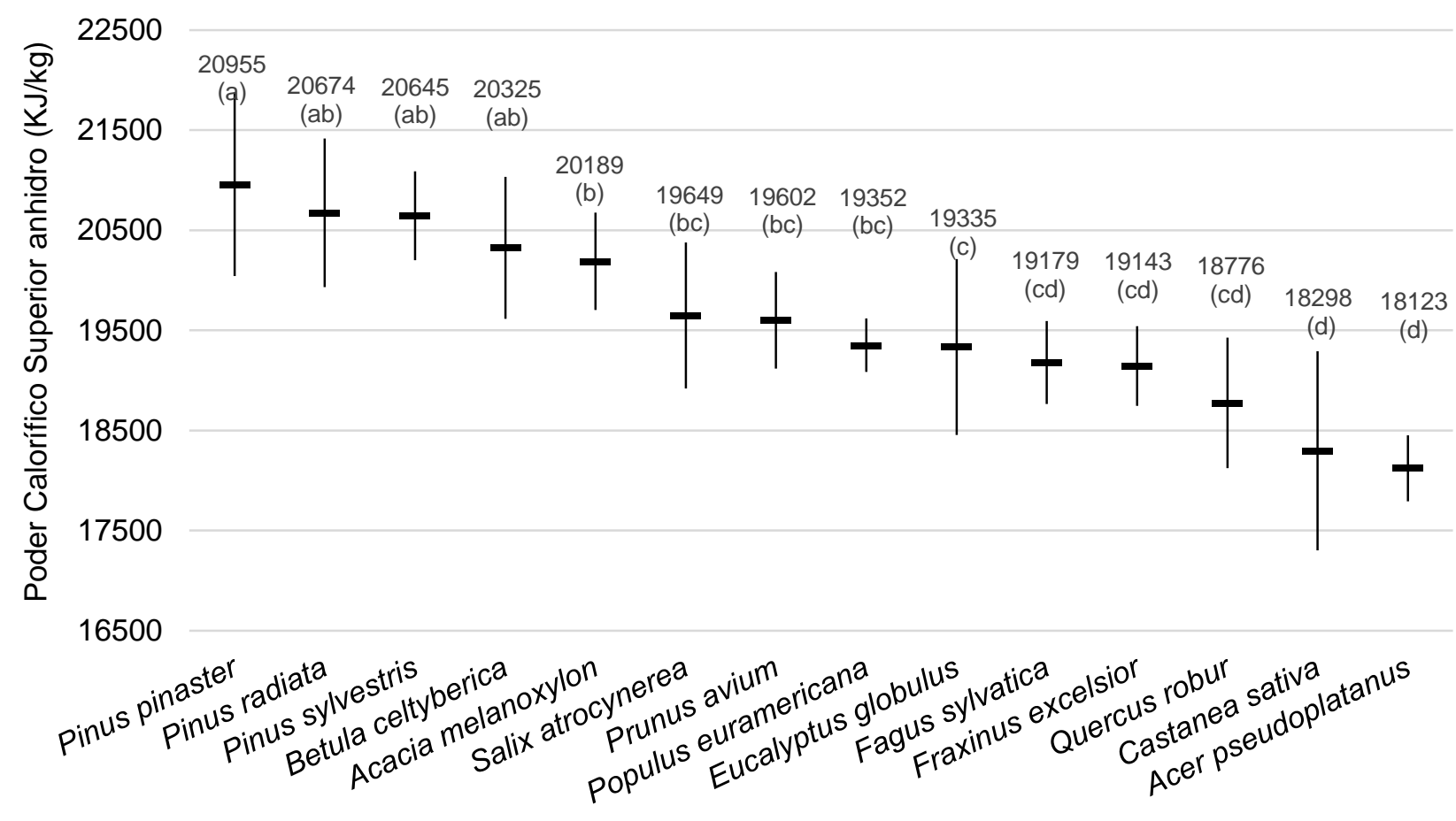

Fig. 1. Poder calorífico superior anhidro $(\mathrm{KJ} / \mathrm{kg})$ promedio e intervalos de confianza de las 14 especies analizadas. 
Tabla 3. Valor promedio y desviación estándar del porcentaje de cenizas a

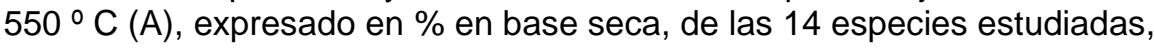
y categoría según norma EN14961

\begin{tabular}{|l|l|l|}
\hline & A (\% b.s.) & $\begin{array}{l}\text { Categoría EN } \\
14961 \\
\text { (astillas y pélets) }\end{array}$ \\
\hline Pinus pinaster & $1.3( \pm 1.2)$ & A2 $(\mathrm{A}<1.5 \%)$ \\
\hline Pinus radiata & $1.3( \pm 1.2)$ & A2 $(\mathrm{A}<1.5 \%)$ \\
\hline Pinus sylvestris & $1.2( \pm 1.1)$ & A2 $(\mathrm{A}<1.5 \%)$ \\
\hline Acacia melanoxylon & $1.2( \pm 0.8)$ & A2 $(\mathrm{A}<1.5 \%)$ \\
\hline Fraxinus excelsior & $1.2( \pm 0.8)$ & A2 $(\mathrm{A}<1.5 \%)$ \\
\hline Betula celtyberica & $1.1( \pm 0.7)$ & A2 $(\mathrm{A}<1.5 \%)$ \\
\hline Prunus avium & $1.1( \pm 0.6)$ & A2 $(\mathrm{A}<1.5 \%)$ \\
\hline Eucalyptus globulus & $1.1( \pm 0.9)$ & A2 $(\mathrm{A}<1.5 \%)$ \\
\hline Fagus sylvatica & $1.1( \pm 0.8)$ & A2 $(\mathrm{A}<1.5 \%)$ \\
\hline Populus euramericana & $1.6( \pm 0.6)$ & B $(\mathrm{A}<3 \%)$ \\
\hline Salix atrocynerea & $1.9( \pm 1.5)$ & $\mathrm{B}(\mathrm{A}<3 \%)$ \\
\hline Quercus robur & $2.2( \pm 2.0)$ & $\mathrm{B}(\mathrm{A}<3 \%)$ \\
\hline Castanea sativa & $1.7( \pm 1.5)$ & $\mathrm{B}(\mathrm{A}<3 \%)$ \\
\hline Acer pseudoplatanus & $2.2( \pm 1.4)$ & $\mathrm{B}(\mathrm{A}<3 \%)$ \\
\hline & & \\
\hline
\end{tabular}

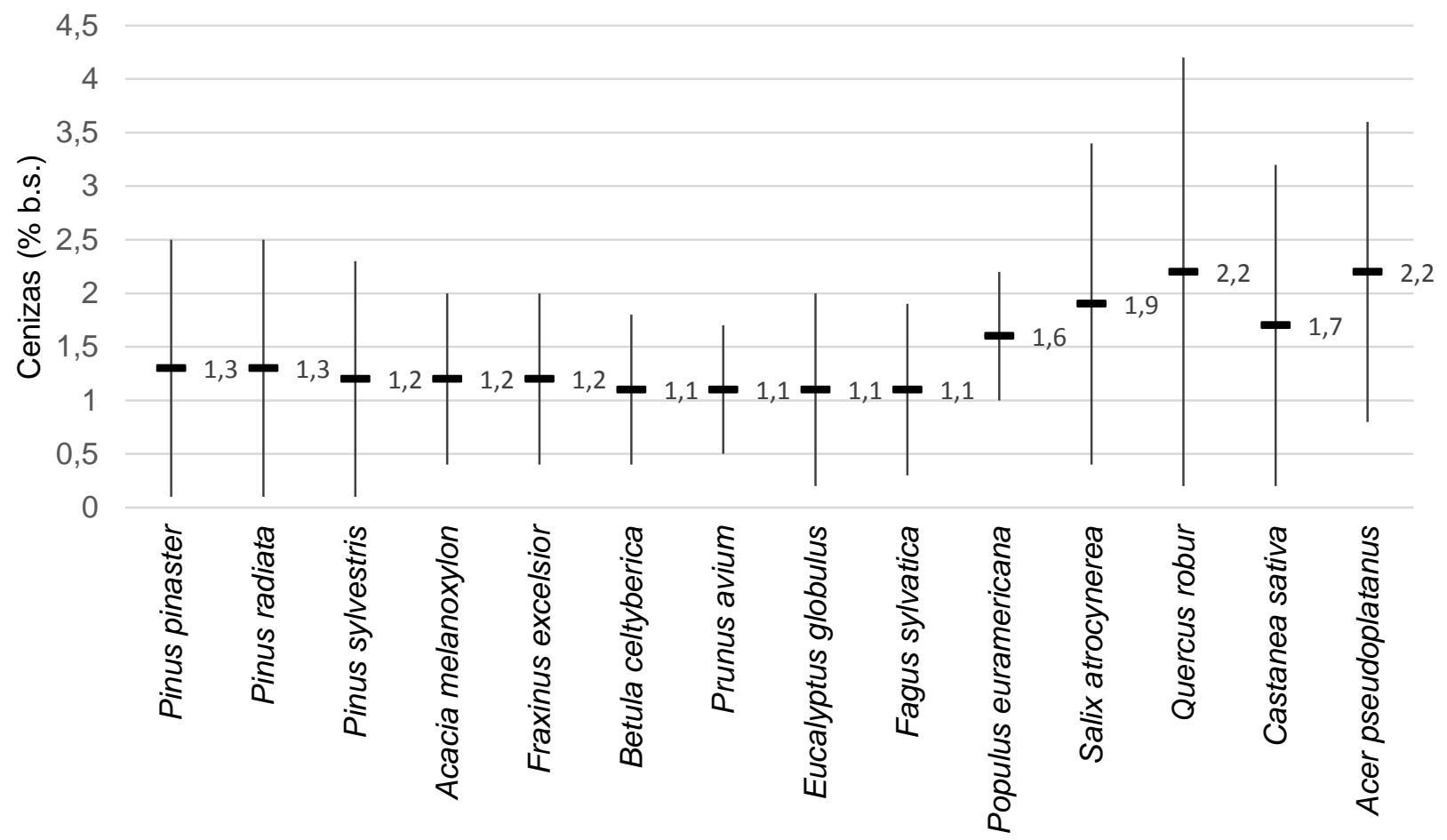

Fig. 2. Contenido de cenizas (porcentaje en base seca) promedio e intervalos de confianza de las especies analizadas.

Los contenidos medios de cenizas medidos fueron superiores al umbral del $0.7 \%$ y del $1 \%$ para la categoría A1 según las normas EN-14961-2 de pélets y EN-14961-4 de astillas, respectivamente. Estos valores más elevados pueden ser explicados por la presencia de corteza en los ramillos y a la presencia de hojas en los residuos de cosecha. Varios autores han notado mayores contenidos de cenizas en las fracciones finas de biomasa forestal residual (hojas y ramillos) (e.g. Nunez-Regueira, 1996, 1997, 1999, 2004, Pérez et al., 2008, García et al. 2012), con valores similares a los observados en el presente estudio, frente a valores más reducidos observados en estudios centrados en madera libre de ramas y hojas (e.g. Lousada et al., 2010, Telmo y Lousada, 2011a, 2011b). 
En base a los contenidos medios de cenizas medidos, las especies estudiadas sólo serían aptas para la comercialización de astillas o pélets de calidad A2 o B, con contenidos inferiores al $1.5 \%$ y $3 \%$, respectivamente. Los mayores contenidos de cenizas se observaron en las frondosas $P$. euramericana, $S$. atrocinerea, $Q$. robur, $C$. sativa y $A$. pseudoplatanus, con contenidos promedios de cenizas superiores al $1.5 \%$. Se debe de considerar además que la alta variabilidad en los contenidos de cenizas hace que, de las 10 muestras analizadas para cada especie, se encontraran muestras por encima de estos umbrales. Así, para las especies señaladas como A2, un $20-30 \%$ de las muestras de cada especie presentaron valores $>1.5 \%$ y $<3 \%$ (categoría $\mathrm{B}$ ). Con respecto a las frondosas de mayor contenido de ceniza arriba señalados, más de la mitad de las muestras analizadas de cada especie presentaron contenidos de cenizas superiores al $1.5 \%$, y entre un 15 y $30 \%$ no serían aptas para la producción de astillas o pélet de calidad B por presentar contenidos superiores al $3 \%$.

El análisis de varianza GLM no detectó diferencias significativas entre los contenidos de cenizas de las especies estudiadas, debido a la alta variabilidad dentro de cada especie (fig. 2 y tabla 3). Estos rangos de variabilidad son similares a los observados en otros estudios de biomasa forestal (e.g. Viana et al., 2010, García et al. 2012), debido a la gran variabilidad presente dentro de cada especie, causada por los distintos contenidos de nutrientes en las distintas fracciones que integran los residuos de cosecha (ramas, hojas) (e.g. Kauter et al., 2004, Pérez et al., 2008, García et al. 2012), así como dentro de cada fracción, por la variabilidad de su composición para cada clase diamétrica, encontrándose mayores contenidos de cenizas en ramillos y troncos más finos debido a su mayor ratio de corteza/madera (e.g. Werkelin et al., 2005, 2011, Vega-Nieva et al., 2010c).

Adicionalmente, los distintos grados de contaminación por suelo son otro de los factores que pueden agravar el contenido de cenizas de la biomasa forestal residual (e.g. Vega-Nieva et al., 2012, 2014). Por último, la fecha de corta y el tiempo que permanecen los residuos en monte, son factores que afectan el contenido de cenizas de los restos forestales (e.g. Kauter et al., 2004, Werkelin et al., 2005, 2011, NunezRegueira, 1996, 1997, 1999, 2004). Un tiempo prolongado de permanencia en monte puede reducir el contenido de nutrientes y cenizas en los residuos al irse depositando las fracciones más finas en el suelo, mejorando la calidad de los residuos como biocombustible y minimizando los nutrientes extraidos (e.g. Ortiz, 2006, Merino et al., 2005), lo cual debe de ser ponderado con el riesgo de incendios de mantener los restos en monte sin triturar (e.g. Stephens y Moghaddas, 2012).

\section{CONCLUSIONES}

Las especies estudiadas mostraron variaciones significativas en su poder calorífico anhidro, encontrándose los mayores valores en especies de coníferas. Estas observaciones corroboran lo reportado por diversos autores en la región de estudio (Elvira y Hernando, 1989; Guillon et al., 1997; Telmo et al., 2010; Lousada et al. 2010; Telmo y Lousada; 2011a 2011b, Garcia et al., 2014a, 2014b) y en varios estudios a nivel global (Demirbas et al., 1997, Channiwala, 2001; Sheng y Azevedo, 2005; Friedl et al., 2005). Estas especies de poder calorífico más elevado serían aptas para la producción de astilla y pélet de calidades A1 en base a su poder calorífico neto, mientras que las frondosas de poder calorífico más reducido, como $Q$. robur, $C$. sativa, A.pseudoplatanus, deberían de ser mezcladas con especies de poder calorífico más elevado para la producción de pélets de calidad EN-Plus.

En base a su contenido de cenizas, los restos forestales de las especies analizadas, serían aptas para la producción de astillas o pélets de categoría A2 o B. Futuros estudios deberían centrarse en analizar el impacto de fechas de corta y tiempo de permanencia de los restos en el monte como factores que potencialmente podrían contribuir a disminuir el contenido de cenizas de los restos de cosecha de destas especies, además de evaluar su impacto sobre la composición de estas cenizas con vistas a minimizar posibles riesgos de fusión o sinterización en las calderas (e.g. Viana et al., 2012, Vega et al., 2010, 2012, 2014).

\section{AGRADECIMIENTOS}

El presente trabajo fue parcialmente financiado por el Proyecto Europeo del VII Marco SilvaPlus.

\section{REFERENCIAS}

Channiwala SA. A unified correlation for estimating HHV of solid, liquid and gaseous fuels, doi:10.1016/S0016-2361(01)00131-4, Fuel 81 1056-1057 (2001)

Demirbas, A. Calculation of higher heating values of biomass fuels, doi:10.1016/S0016-2361(97)85520-2, Fuel, 76 (5): 431-434 (1997) 
Dopazo, R.; Vega-Nieva, D.; Ortiz, L. El aprovechamiento de biomasa residual y de cultivos energéticos: experiencias recientes. 5o Congreso Forestal Español. Ávila, 21-25 de Septiembre de (2009)

Dopazo, R.; Vega-Nieva, D.; Ortiz, El aprovechamiento de la biomasa en cultivos energéticos leñosos: una nueva oportunidad para la valorización del monte. I Congreso Internacional de Valorización Integral do Monte, Santiago de Compostela del 19 al 22 de Noviembre de (2008)

Elvira, L.M.; Hernando, C., Inflamabilidad y energía de las especies de sotobosque: estudio piloto con aplicación a los incendios forestales, Monografías INIA. (1989)

Fernández-Puratich, H., Oliver-Villanueva, J.V., Cuantificación de biomasa y valor energético de renovales de Quercus ilex en condiciones mediterráneas, 2014, ISSN: 0304-8799, Bosque 35, 65-74 (2014).

Friedl A, Padouvas E, Rotter H, Varmuza K., Prediction of heating values of biomass fuel from elemental composition, doi:10.1016/j.aca.2005.01.041, Anal ChimActa 544,191-8 (2005).

García, R.; Pizarro, C.; Lavín, A.G.; Bueno, J.L., Characterization of Spanish biomass wastes for energy use, doi:10.1016/j.biortech.2011.10.004, Bioresource Technology 103,249-258 (2012).

García-Ubaque, César A, Vaca-Bohórquez, Martha L and Talero, Gabriel F Aprovechamiento de Biomasa Peletizada en el Sector Ladrillero en Bogotá-Colombia: Análisis Energético y Ambiental, ISSN 0718-0764, Inf. Tecnol 24(3), 115-120 (2013).

García,R., Pizarro C., Lavín, A.G., Bueno J. L., Spanish biofuels heating value estimation. Part I: Ultimate analysis data, doi:10.1016/j.fuel.2013.08.048, Fuel 117, 1130-1138 (2014a)

García,R., Pizarro C., Lavín, A.G., Bueno J. L., Spanish biofuels heating value estimation. Part II: Proximate analysis data, doi:10.1016/j.fuel.2013.08.049, Fuel 117, 1139-1147 (2014b)

Gillon, D.; Hernando, C.; Valette, J.C.; Joffre, R., Fast estimation of the calorific values of forest fuels by near-infrared reflectance spectroscopy, doi:10.1139/x97-021, Canadian Journal of Forest Research 27, 760765 (1997)

Gómez, Elkin A, Ríos, Luis A and Peña, Juan D, Efecto del Pretratamiento de Biomasa Maderera en el Rendimiento a Etanol, ISSN 0718-0764, Inf. Tecnol. 24(5), 113-122 (2013)

Gómez, Elkin A, Ríos, Luis A and Peña, Juan D Madera, un Potencial Material Lignocelulósico para la Producción de Biocombustibles en Colombia, ISSN 0718-0764, Inf. tecnol. 23(6), 73-86. (2012)

Kauter, D.; Lewandowski, I.; Claupeina, W., Quantity and quality of harvestable biomass from Populus short rotation coppice for solid fuel use-a review of the physiological basis and management influences, doi:10.1016/S0961-9534(02)00177-0, Biomass and Bioenergy, 24, 411- 427 (2003)

Lousada, J.;Telmo, C.; Moreira, N., Proximate analysis, backwards stepwise regression between gross calorific value, ultimate and chemical analysis of wood, doi:10.1016/j.biortech.2010.01.021, BioresourceTecnology 101(11), 3808-3815 (2010)

Madrigal, J.; Guijarro, M; Hernando, C; Díez, C; Marino, E., Effective Heat of Combustion for Flaming Combustion of Mediterranean Forest Fuels, doi:10.1007/s10694-010-0165-x, Fire Technology 47,461-474 (2011)

Mendoza, Jorge M., Análisis Exergético de la Gasificación de Biomasa, ISSN 0718-0764, Inf. tecnol. 23(5): 85-96 (2012)

Merino A., Balboa M.A., Rodríguez Soalleiro R., Alvarez J.G., Nutrient exports under different harvesting regimes in fast-growing forest plantations in southern Europe, doi:10.1016/j.foreco.2004.10.074, Forest Ecology and Management 207 325-339 (2005)

Nuñez Regueira, L., Calorific values and flammability of forest species in Galicia. Coastal and hillsidezone, doi:10.1016/S0960-8524(96)00083-1, BioresourceTecnology 57,283-289 (1996)

Nuñez Regueira, L., Calorific values and flammability of forest species in Galicia. Continental high mountainous and sumid atlantic zones, doi:10.1016/S0960-8524(97)00053-9, BioresourceTecnology 61,111119 (1997)

Núñez-Regueira, L.; Proupín-Castiñeiras, J.; Rodríguez-Añón, J.A., Energy evaluation of forest residues originated from shrub species in Galicia, doi:10.1016/S0960-8524(03)00169-X, Bioresource Technology 91, 215-221 (2004)

Núñez-Regueira, L.; Rodríguez, J.; Proupín, J; Mouriño, B., Forest waste as an alternative energy source, doi:10.1016/S0040-6031(98)00630-3, Thermochimica Acta 328, 105-110 (1999)

Ortiz Torres, L. 2006. Xiloenergética. Ed. Gamesal. Vigo, (2006) 
Pérez, S.; Renedo, C.J.; Ortiz, A.; Manana, M., Energy potential of waste from 10 forest species in the North of Spain (Cantabria), doi:10.1016/j.biortech.2007.12.014, Bioresource Technology 99, 633-645 (2008)

Stephens SL, Moghaddas JJ, Experimental fuel treatment impacts on forest structure, potential fire behavior, and predicted tree mortality in a California mixed conifer forest, doi: 10.1016/j.foreco.2005.03.070, For Ecol Manage 215, 21-36 (2005)

Sheng C, Azevedo JLT. , Estimating the higher heating value of biomass fuels from basic analysis data, doi:10.1016/j.biombioe.2004.11.008, Biomass Bioenergy 28, 499-507 (2005)

Telmo C, Lousada J, Moreira N., Proximate analysis, backwards stepwise regression between gross calorific value, ultimate and chemical analysis of wood, doi:10.1016/j.biortech.2010.01.021, Bioresource Technoly 101:3808-15 (2010)

Telmo, C.; Lousada, J., Heating values of wood pellets from different species, doi:10.1016/j.biombioe.2011.02.043, Biomass and bioenergy 35, 2634-2639 (2011a)

Telmo, C.; Lousada, J., The explained variation by lignin and extractive contents on higher heating value of wood, doi:10.1016/j.biombioe.2010.12.038, Biomass and bioenergy,35: 1663-1667 (2011b)

UNE, Una Norma Española, aprobada por la Asociación Española de Normalización y Certificación (AENOR) (2010)

UNE, Una Norma Española, aprobada por la Asociación Española de Normalización y Certificación (AENOR) (2012)

UNE, Una Norma Española, aprobada por la Asociación Española de Normalización y Certificación (AENOR) (2013)

Vega-Nieva, D.J.; Dopazo, R; Ortiz, L., Slagging risk of mediterranean biomasses for combustion, In: World Bioenergy, 25-27 May, Jönköping, Sweden (2010a)

Vega-Nieva, D.J.; Dopazo, R; Ortiz, L., Manual de Cultivos Energéticos. Cátedra ENCE. Ed. Gamesal. 209 pg. ISBN 678-84-95046-45-1 (2010b)

Vega-Nieva, D.J.; Dopazo, R; Ortiz, L., Aspectos Medioambientales del aprovechamiento de la biomasa y cultivos energéticos, I Congreso Ibérico de Biocombustibles sólidos. 21-23 Junio 2010, Pontevedra, España. (2010c)

Vega-Nieva, D.; Dopazo, R.; Ortiz, L., Strategies for minimizing ash slagging in combustion of mediterranean biomasses. World Bioenergy 2012. 29-31 May Jönköping, Suecia. (2012)

Vega-Nieva, D.; Alvarez, C., Ortiz, L., Results of new laboratory methods and slagging classification systems for the prediction and quantification of ash slagging in woody and herbaceous biomass fuels. Central European Biomass Conference. Graz. Aunstria. January 2014. (2014)

Viana, H.; Cohen, W.B.; Lopes, D.; Aranha, J., Assessment of forest biomass for use as energy. GIS-based analysis of geographical availability and locations of wood-fired power plants in Portugal, doi:10.1016/j.apenergy.2010.02.007, Applied Energy 87, 2551-2560 (2010)

Viana, H.; Vega-Nieva, D.J.; Ortiz Torres, L.; Lousada, J.; Aranha, J. Fuel characterization and biomass combustion properties of selected native woody shrub species from central Portugal and NW Spain, doi:10.1016/j.fuel.2012.06.035, Fuel 102, 737-745 (2012)

Werkelin, J., Skrifvars, B.J., Hupa, M., Ash-forming elements in four Scandinavian wood species part 1: Summer harvest, doi:10.1016/j.biombioe.2005.06.005, Biomass and Bioenergy 29, 451-466 (2005)

Werkelin, J., Lindberg, D., Boström, D., Skrifvars, B.J., Hupa, M., Ash-forming elements in four Scandinavian wood species part 3: Combustion of five spruce samples, doi:10.1016/j.biombioe.2010.10.010, Biomass and Bioenergy 35(1), 725-733 (2011) 
